\title{
THYRISTOR CONTROLLED PHASE SHIFTER BASED STABILIZER DESIGN USING SIMULATED ANNEALING ALGORITHM
}

\author{
M. A. Abido
}

\author{
Electrical Engineering Department \\ King Fahd University of Petroleum and Minerals \\ Dhahran 31261, Saudi Arabia \\ E-mail: mabido@kfupm.edu.sa
}

\begin{abstract}
This paper presents a thyristor controlled phase shifter (TCPS) based stabilizer design using simulated annealing (SA) algorithm. The design problem of TCPS based stabilizer is formulated as an optimization problem. An eigenvalue based objective function to increase the system damping is proposed. Then, SA algorithm is employed to search for optimal setting of stabilizer parameters. Two different control schemes have been proposed and tested on a weakly connected power system with different disturbances and loading conditions. Nonlinear simulation results show the potential of SA algorithm to the design problem of TCPS based stabilizer. Effectiveness of the proposed control schemes over a wide range of loading conditions has been demonstrated. It was also observed that the TCPS controller provides efficient damping of low frequency oscillations and improves greatly the voltage profile of the system under severe disturbances.
\end{abstract}

Keywords: thyristor controlled phase shifter, power system stabilizer, simulated annealing algorithm.

\section{INTRODUCTION}

Due to increasing complexity of electrical power systems, there has been increasing interest in the stabilization of such systems. In the past two decades, the utilization of supplementary excitation control signals for improving the dynamic stability of power systems and damping out the low frequency oscillations has received much attention [1-3]. Nowadays, the conventional power system stabilizer (CPSS) - a fixed parameters lead-lag compensator - is widely used by power system utilities [1]. Although PSSs extend the power system stability limit by enhancing the system damping, they suffer a drawback of being liable to cause great variations in the voltage profile and they may even result in leading power factor operation and losing system stability under severe disturbances [4-5].

The recent advances in power electronics have led to the development of the flexible alternating current transmission systems (FACTS). FACTS are designed to overcome the limitations of the present mechanically

Paper BPT99-457-12 accepted for presentation at the IEEE Power Tech'99 Conference, Budapest, Hungary, Aug 29 - Sep 2, 1999. controlled power systems and enhance power system stability by using reliable and high-speed electronic devices. One of the promising FACTS devices is the thyristor controlled phase shifter (TCPS). A considerable attention has been directed to analysis and realization of various TCPS schemes [6-7]. However, a relatively little work in TCPS control aspects has been reported in the literature. Edris [8] proposed a simple control algorithm based on equal area criterion. Recently, Jiang et al [9] proposed a control technique based on nonlinear variable structure control theory. In their control scheme the phase shift angle is determined as a nonlinear function of rotor angle and speed. However, in real life power system with a large number of generators, the rotor angle of a single generator measured with respect to the system reference will not be very meaningful. Tan and Wang [10] proposed a direct feedback linearization technique to linearize and decouple the power system model to design the excitation and phase shifter controllers.

Despite the potential of modern control techniques with different structures, power system utilities still prefer a conventional lead-lag controller structure [2-3]. The reasons behind that might be the ease of on-line tuning and the lack of assurance of the stability related to some adaptive or variable structure techniques. It is shown that the appropriate selection of conventional lead-lag stabilizer parameters results in effective damping to low frequency oscillations [3].

In the last few years, simulated annealing (SA) algorithm [11-12] appeared as a promising heuristic algorithm for handling the combinatorial optimization problems. It has been theoretically proved that the SA algorithm converges to the optimum solution [11]. Another strong feature of SA algorithm is that a complicated mathematical model is not needed and the constraints can be easily incorporated [11].

In this paper, design of TCPS based stabilizer using SA algorithm is investigated. Two different control schemes have been proposed and tested on a weakly connected power system. Based on eigenvalue analysis and simulation results, it was observed that the proposed control schemes provide good damping of electromechanical modes of oscillations, enhance power system stability, and improve the system voltage profile. 
In this study, a single machine infinite bus system with an TCPS shown in Fig. 1 is considered. The TCPS has a ratio of $1: 1 /-\Phi$. The generator is connected to the infinite bus via a transmission line. The configuration of an TCPS and the corresponding phasors of the system voltages are shown in Fig. 2. Generally, TCPS is a device that changes the relative phase angle between system voltages. Therefore, the real power flow can be regulated to mitigate the low frequency oscillations and enhance power system transient stability. To achieve this goal, the system voltage $v$ is provided to the converter by the exciting transformer ET as shown in Fig. 2. The converter output voltage $v_{\text {TCPS }}$ is injected in the system by the series boosting transformer BT. The converter controls the magnitude and/or the angle of the injected voltage [11]. As shown in the phasor diagram, the voltage at the end bus of TCPS $v$ ' is phase shifted with respect to the input voltage $v$ by angle $\Phi$. Therefore, the real power flow can be described as

$$
P_{e}=\frac{E_{q}^{\prime} v_{b}}{X} \sin (\delta-\Phi)
$$

Hence, controlling the angle $\Phi$ can regulate the real power flow to mitigate the low frequency oscillations.

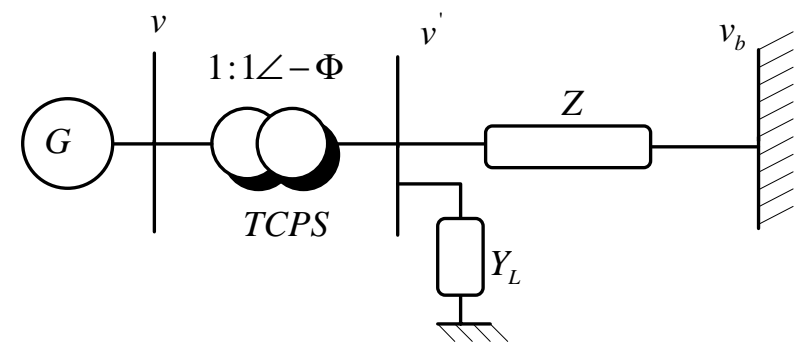

Fig. 1: Single machine infinite bus system with a TCPS

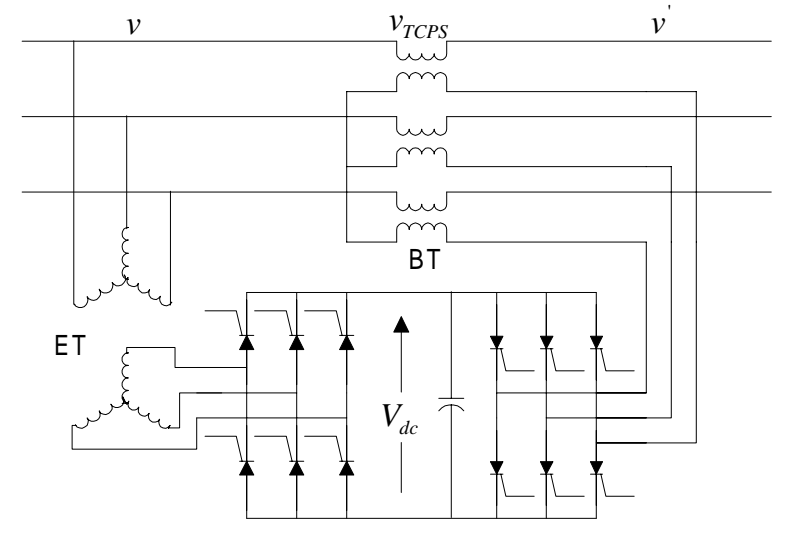

Fig. 2: The configuration of TCPS

\section{LINEARIZED POWER SYSTEM MODEL}

The generator is represented by the third-order model comprising of the electromechanical swing equation and the generator internal voltage equation. The swing equation is divided to the following equations

$$
\rho \delta=\omega_{b}(\omega-1)
$$

$$
\rho \omega=\left(P_{m}-P_{e}-D(\omega-1)\right) / M
$$

$P_{m}$ is assumed to be constant and $P_{e}$ can be expressed as

$$
P_{e}=v_{d} i_{d}+v_{q} i_{q}
$$

The internal voltage, $E_{q}^{\prime}$, equation is

$$
\rho E_{q}^{\prime}=\left(E_{f d}-\left(x_{d}-x_{d}^{\prime}\right) i_{d}-E_{q}^{\prime}\right) / T_{d o}^{\prime}
$$

The IEEE Type-ST1 excitation system shown in Fig. 3 is considered in this study. It can be described as

$$
\rho E_{f d}=\left(K_{A}\left(V_{r e f}-v+u_{P S S}\right)-E_{f d}\right) / T_{A}
$$

where;

$$
\begin{aligned}
& v=\left(v_{d}^{2}+v_{q}^{2}\right)^{1 / 2} \\
& v_{d}=x_{q} i_{q} \\
& v_{q}=E_{q}^{\prime}-x_{d}^{\prime} i_{d}
\end{aligned}
$$

Fig. 4 illustrates the block diagram of an TCPS stabilizer. The Phase shift angle of the TCPS, $\Phi$, is expressed as

$$
\rho \Phi=\left(K_{s}\left(\Phi_{r e f}-u_{T C P S}\right)-\Phi\right) / T_{s}
$$

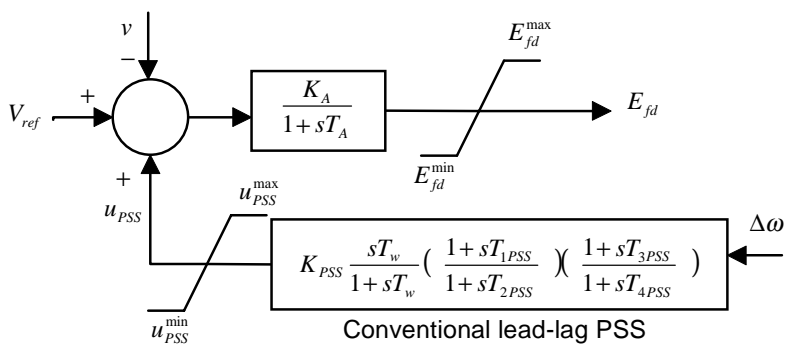

Fig. 3: IEEE Type-ST1 excitation system with conventional PSS

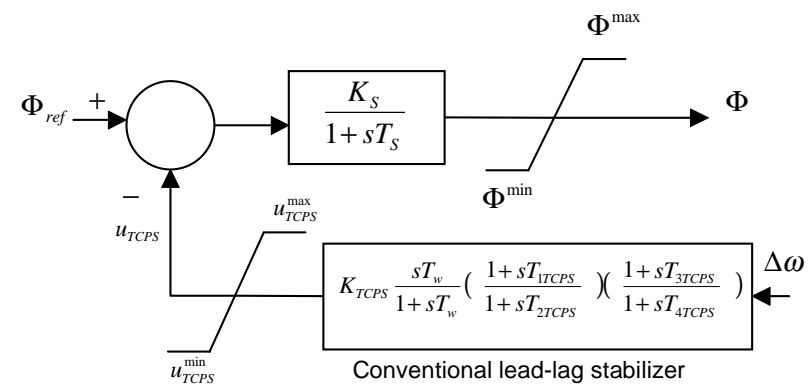

Fig. 4: TCPS with conventional lead-lag stabilizer

In the stabilizer design problem, the linearized incremental model around a nominal operating point is usually employed [1-3]. Generally, the linearized system model can be written as

$$
\rho X=A X+B U
$$

Here, the state vector $X$ is $\left[\Delta \delta, \Delta \omega, \Delta E_{q}^{\prime}, \Delta E_{f d}\right]^{T}$ and the control vector $U$ is $\left[u_{P S S}, \Delta \Phi\right]^{T}$. 
A TCPS based stabilizer and PSS as shown in Figs. 3 and 4 are considered in this study. In this structure, the washout time constant $T_{w}$ and the time constants $T_{2}$ TсPS, $T_{4}$ TCPS, $T_{2}$ PSS, and $T_{4}$ PSS are usually prespecified. In this study, $T_{w}=5 \mathrm{~s}$ and $T_{2} \quad$ TCPS $=T_{4}$ TCPS $=T_{2}$ PSS $=T_{4} \quad$ PSS $=0.1 \mathrm{~s}$. The stabilizer gains $K_{\mathrm{TCPS}}$ and $K_{\mathrm{PSS}}$, and time constants $T_{1 \text { TCPS, }}$ $T_{3}$ TCPS, $T_{1 \text { PSS }}$, and $T_{3}$ PSS are remained to be determined. The following control schemes are proposed.

- Scheme (a) where TCPS based stabilizer only is considered. In this scheme, the tuning parameters are $K_{\mathrm{TCPS}}, T_{1 \text { TCPS}}$, and $T_{3}$ TCPS.

- Scheme (b) where coordinated design of both TCPS based stabilizer and PSS is considered. In this scheme, the tuning parameters are $K_{\mathrm{TCPS}}, T_{1 \mathrm{TCPS}}, T_{3}$ TCPS,$K_{\mathrm{PSS}}$, $T_{1 \text { PSs, }}$, and $T_{3 \text { PSs. }}$

Participation factors method [13] is used to identify the eigenvalues of the system matrix $A$ associated with the electromechanical modes. The following objective function $J$ to increase the system damping is proposed as

$$
J=\zeta \text { of electromechanical mode }
$$

where $\zeta$ is the damping ratio. The problem constraints are the optimized parameter bounds. Therefore, the design problem can be formulated as the following optimization problem.

\section{Maximize $J$}

\section{Subject to}

$$
\begin{aligned}
& K_{\mathrm{TCPS}}{ }^{\min } \leq K_{\mathrm{TCPS}} \leq K_{\mathrm{TCPS}}{ }^{\max } \\
& T_{1 \text { TCPS }}{ }^{\text {in }} \leq T_{1 \text { TCPS }} \leq T_{1 \text { TCPS }}^{\max } \\
& T_{3 \text { TCPS }}{ }^{\text {in }} \leq T_{3 \text { TCPS }} \leq T_{3 \text { TCPS }}{ }^{\max } \\
& K_{\mathrm{PSS}}^{{ }^{\text {min }}} \leq K_{\mathrm{PSS}} \leq K_{\mathrm{PSS}}{ }^{\max } \\
& \text { (17) } \\
& T_{1 \text { PSS }}{ }^{\text {min }} \leq T_{1 \text { PSS }} \leq T_{1 \text { PSS }}{ }^{\max } \\
& T_{3 \text { PSS }}{ }^{\text {min }} \leq T_{3 \text { PSS }} \leq T_{3 \text { PSS }}{ }^{\text {max }}
\end{aligned}
$$

The proposed approach employs SA algorithm to solve this optimization problem and search for optimal or near optimal set of the optimized parameters.

\section{SIMULATED ANNEALING ALGORITHM}

\subsection{Overview}

Simulated annealing is an optimization technique that simulates the physical annealing process in the field of combinatorial optimization. Annealing is the physical process of heating up a solid until it melts, followed by slow cooling it down by decreasing the temperature of the environment in steps. At each step, the temperature is maintained constant for a period of time sufficient for the solid to reach thermal equilibrium.

Metropolis et al [12] proposed a Monte Carlo method to simulate the process of reaching thermal equilibrium at a fixed value of the temperature $T$. In this method, a randomly generated perturbation of the current configuration of the solid is applied so that a trial configuration is obtained. This trial configuration is accepted and becomes the current configuration if it satisfies an acceptance criterion. The process continues until the thermal equilibrium is achieved after a large number of perturbations. By gradually decreasing the temperature $T$ and repeating Metropolis simulation, new lower energy levels become achievable. As $T$ approaches zero least energy configurations will have a positive probability of occurring.

\subsection{SA Algorithm}

At first, the analogy between a physical annealing process and a combinatorial optimization problem is based on the following [11]:

- Solutions in an optimization problem are equivalent to configurations of a physical system.

- The cost of a solution is equivalent to the energy of a configuration.

In addition, a control parameter $C_{p}$ is introduced to play the role of the temperature $T$.

The basic elements of SA are defined as follows :-

- Current, trial, and best solutions, $x_{\text {current, }} x_{\text {trial }}$, and $x_{\text {best }}$ : these solutions are sets of the optimized parameter values at any iteration.

- Acceptance criterion: at any iteration, the trial solution can be accepted as the current solution if it meets one of the following critera; (a) $J\left(x_{\text {trial }}\right)<$ $J\left(x_{\text {current }}\right)$; (b) $J\left(x_{\text {trial }}\right)>J\left(x_{\text {current }}\right)$ and $\exp \left(-\left(J\left(x_{\text {trial }}\right)-\right.\right.$ $\left.\left.J\left(x_{\text {current }}\right)\right) / C_{p}\right) \geq \operatorname{rand}(0,1)$. Here, $\operatorname{rand}(0,1)$ is a random number with domain $[0,1]$ and $J\left(x_{\text {trial }}\right)$ and $J\left(x_{\text {current }}\right)$ are the objective function values associated with $x_{\text {trial }}$ and $x_{\text {current }}$ respectively. Criterion (b) indicates that the trial solution is not necessarily rejected if its objective function is not as good as that of the current solution with hoping that a much better solution become reachable.

- Acceptance ratio: at a given value of $C_{p}$, an $n_{1}$ trial solutions can be randomly generated. Based on the acceptance criterion, an $n_{2}$ of these solutions can be accepted. The acceptance ratio is defined as $n_{2} / n_{1}$.

- Cooling schedule: it specifies a set of parameters that governs the convergence of the algorithm. This set includes an initial value of control parameter $C_{p 0}$, a decrement function for decreasing the value of $C_{p}$, and a finite number of iterations or transitions at each value of $C_{p}$, i.e. the length of each homogeneous Markov chain. The initial value of $C_{p}$ should be large enough to allow virtually all transitions to be accepted. However, this can be achieved by starting off at a small value of $C_{p 0}$ and multiplying it with a constant larger than $1, \alpha$, i.e. $C_{p 0}=\alpha C_{p 0}$. This process continues until the acceptance ratio is close to 1 . This is equivalent to heating up process in physical systems. The decrement function for decreasing the value of $C_{p}$ is given by $C_{p}=\mu C_{p}$ where $\mu$ is a constant smaller than but close to 1 . Typical values lie between 0.8 and 0.99 [11].

- Equilibrium condition: it occurs when the current solution does not change for a certain number of 
iterations at a given value of $C_{p}$. It can be achieved by generating a large number of transitions at that value of $C_{p}$.

- Stopping Criteria: these are the conditions under which the search process will terminate. In this study, the search will terminate if one of the following criteria is satisfied: (a) the number of Markov chains since the last change of the best solution is greater than a prespecified number; or, (b) the number of Markov chains reaches the maximum allowable number.

The SA algorithm can be described in steps as follows:

Step 1: Set the initial value of $C_{p 0}$ and randomly generate an initial solution $x_{\text {initial }}$ and calculate its objective function. Set this solution as the current solution as well as the best solution, i.e. $x_{\text {initial }}=x_{\text {current }}=x_{\text {best }}$.

Step 2: Randomly generate an $n_{1}$ of trial solutions in the neighborhood of the current solution.

Step 3: Check the acceptance criterion of these trial solutions and calculate the acceptance ratio. If acceptance ratio is close to 1 go to step 4; else set $C_{p 0}=\alpha C_{p 0}, \alpha>1$, and go back to step 2 .

Step 4: Set the chain counter $k_{c h}=0$.

Step 5: Generate a trial solution $x_{\text {trial }}$. If $x_{\text {trial }}$ satisfies the acceptance criterion set $x_{\text {current }}=x_{\text {trial }}, J\left(x_{\text {current }}\right)=$ $J\left(x_{\text {trial }}\right)$, and go to step 6; else go to step 6 .

Step 6: Check the equilibrium condition. If it is satisfied go to step 7; else go to step 5 .

Step 7: Check the stopping criteria. If one of them is satisfied then stop; else set $k_{c h}=k_{c h}+1$ and $C_{p}=\mu C_{p}$, $\mu<1$, and go back to Step 5 .

6

\section{APPLICATION OF SA TO THE DESIGN PROBLEM}

SA algorithm has been applied to search for optimal settings of the optimized parameters of the proposed control schemes. The final settings of the optimized parameters for the proposed scheme (a) and (b) are given in Tables 1 and 2 respectively. The convergence rate of the objective function $J$ with the number of iterations is shown in Fig. 5.

It is worth mentioning that the optimization process has been carried out with the system operating at nominal loading condition given below in Table 3 .

TABLE 1: THE OPTIMAL SETTINGS OF THE STABILIZER PARAMETERS OF THE PROPOSED SCHEME (a)

\begin{tabular}{ccc}
\hline$K_{\mathrm{TCPS}}$ & $T_{1 \mathrm{TCPS}}$ & $T_{3 \mathrm{TCPS}}$ \\
\hline 96.99 & 0.080 & 0.085 \\
\hline
\end{tabular}

TABLE 2: THE OPTIMAL SETTINGS OF THE STABILIZER PARAMETERS OF THE PROPOSED SCHEME $(\mathrm{b})$

\begin{tabular}{cccccc}
\hline$K_{\mathrm{TCPS}}$ & $T_{1 \mathrm{TCPS}}$ & $T_{3 \mathrm{TCPS}}$ & $K_{\mathrm{PSS}}$ & $T_{1 \mathrm{PSS}}$ & $T_{3 \mathrm{PSS}}$ \\
\hline 8.760 & 0.944 & 0.119 & 40.62 & 0.150 & 0.115 \\
\hline
\end{tabular}

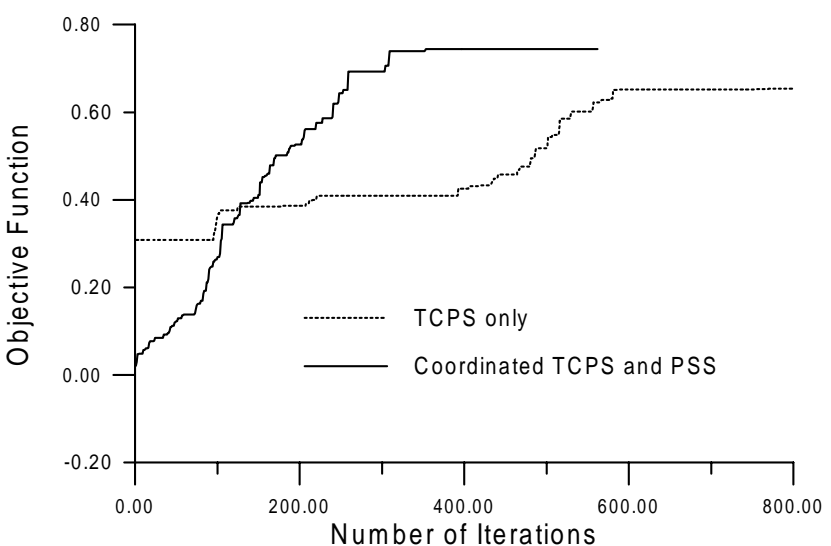

Fig. 5: Objective function variations of the proposed schemes

\section{SIMULATION RESULTS}

To assess the effectiveness and robustness of the proposed control schemes, three different loading conditions given in Table 3 were considered. Moreover, different disturbances and system parameter variations were applied. The performance of the proposed control schemes is compared to that of CPSS given in [14].

It is worth mentioning that all the time domain simulations were carried out using the nonlinear power system model. The system data is given in [15].

\subsection{Nominal Loading}

At this loading condition, the system eigenvalues with and without the proposed control schemes are given in Table 4. It is shown that the open loop system is unstable and the CPSS [14] stabilizes the system with an electromechanical mode damping ratio $\zeta$ of 0.254 . The corresponding damping ratios of the proposed schemes (a) and (b) are 0.656 and 0.744 respectively. It is quite clear that the proposed control schemes enhance greatly the damping of electromechanical mode of oscillation. However, better damping can be achieved with proper coordinated design of TCPS based stabilizer and PSS.

TABLE 3: LOADING CONDITIONS

\begin{tabular}{ccccc}
\hline Loading & $P(\mathrm{pu})$ & $Q(\mathrm{pu})$ & $v(\mathrm{pu})$ & $\Phi($ Deg. $)$ \\
\hline Nominal & 1.0 & 0.015 & 1.05 & 0.0 \\
Leading PF & 0.70 & -0.20 & 1.05 & 5.0 \\
Heavy & 1.10 & 0.40 & 1.05 & 5.0 \\
\hline
\end{tabular}

TABLE 4: SYSTEM EIGENVALUES WITH AND WITHOUT CONTROL

\begin{tabular}{cccc}
\hline No PSS & CPSS [7] & Prop. Sch. (a) & Prop. Sch. (b) \\
\hline$+0.3 \pm \mathrm{j} 5.0^{*}$ & $-1.2 \pm \mathrm{j} 4.4^{*}$ & $-3.2 \pm \mathrm{j} 3.7^{*}$ & $-4.0 \pm \mathrm{j} 3.5^{*}$ \\
$-10.4 \pm \mathrm{j} 3.3$ & $-4.6 \pm \mathrm{j} 7.4$ & $-6.6 \pm \mathrm{j} 7.6$ & $-6.3 \pm \mathrm{j} 5.6$ \\
--------- & $-0.2,-18.7$ & $-11.2 \pm \mathrm{j} 0.9$ & $-10 \pm \mathrm{j} 0.01$ \\
-------- & -------- & $-0.2,-18.1$ & $-15.2 \pm \mathrm{j} 8.1$ \\
------- & ------- & $-9.2,-0.2,-0.2$ \\
\hline
\end{tabular}

Eigenvalues associated with the electromechanical mode 
The behavior of the proposed control schemes under transient conditions was verified by applying a three phase fault at the infinite bus at $t=1 \mathrm{~s}$. The fault duration was $0.1 \mathrm{~s}$. The system response is shown in Figs. 6 and 7. It can be seen that the first swing in the torque angle is significantly suppressed with the proposed control schemes. Thus, TCPS based stabilizer outperforms CPSS in damping of first swing and increasing of stability margin. In Fig. 7, the voltage profile is greatly improved with the proposed TCPS based stabilizer when applied independently. This confirms that PSS degrades the voltage profile under severe disturbances.

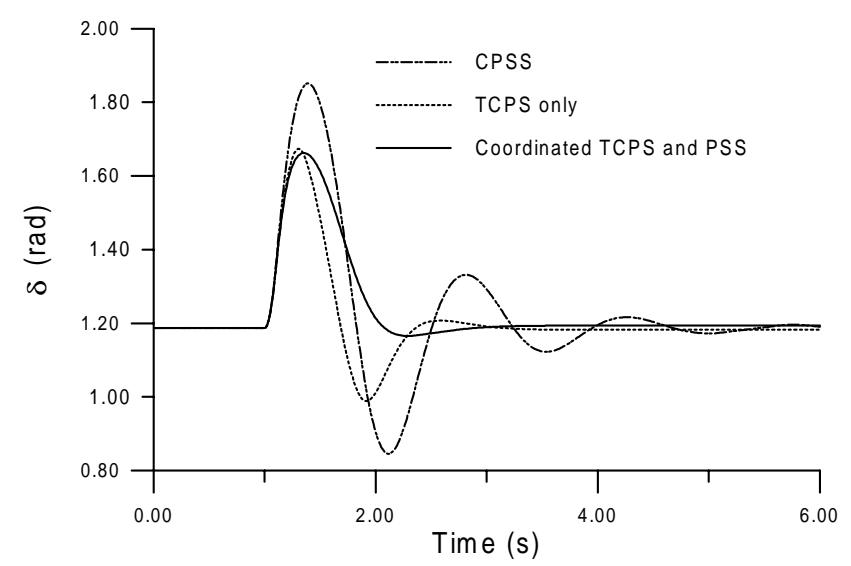

Fig. 6: $\delta$ response with nominal loading

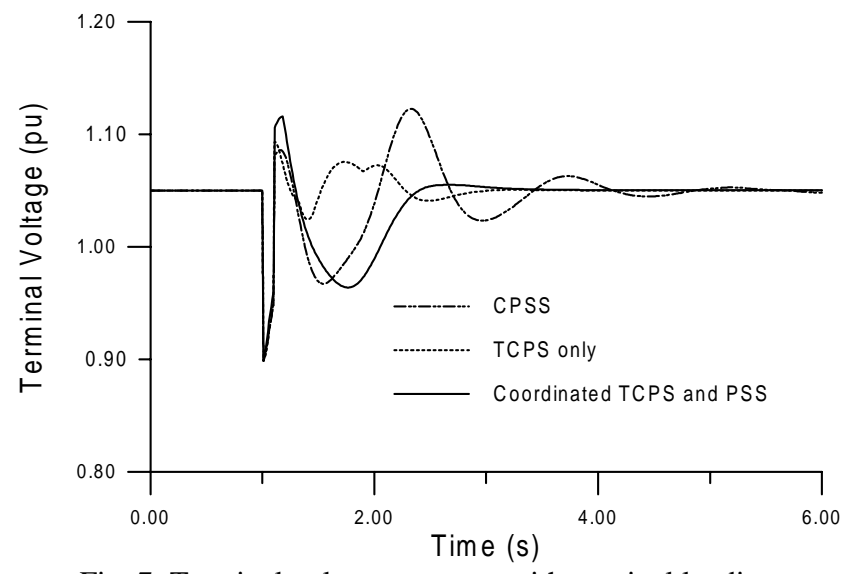

Fig. 7: Terminal voltage response with nominal loading

\subsection{Leading PF Loading}

It may become necessary to operate the generator at a leading power factor. In this case, the stability margin is reduced and it becomes important to test the proposed control schemes under this situation. A three phase fault test has been applied at the infinite bus for $0.1 \mathrm{~s}$. The system response is shown in Figs. 8 and 9. It is shown that the system damping characteristics are significantly enhanced with the proposed control schemes.

\subsection{Heavy Loading}

A three phase fault disturbance at the infinite bus for 0.05s was applied. The results are shown in Figs. 10 and 11. It can be seen that the proposed control schemes suppress the first swing in torque angle and extend the system stability limit. In addition, the voltage profile is greatly improved with the proposed TCPS based stabilizer in terms of overshoots and settling time.

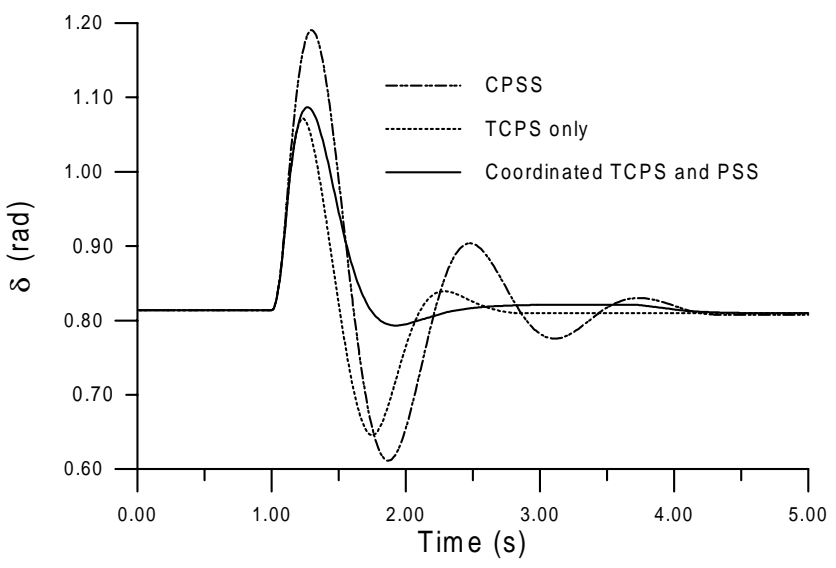

Fig. 8: $\delta$ response with leading PF loading

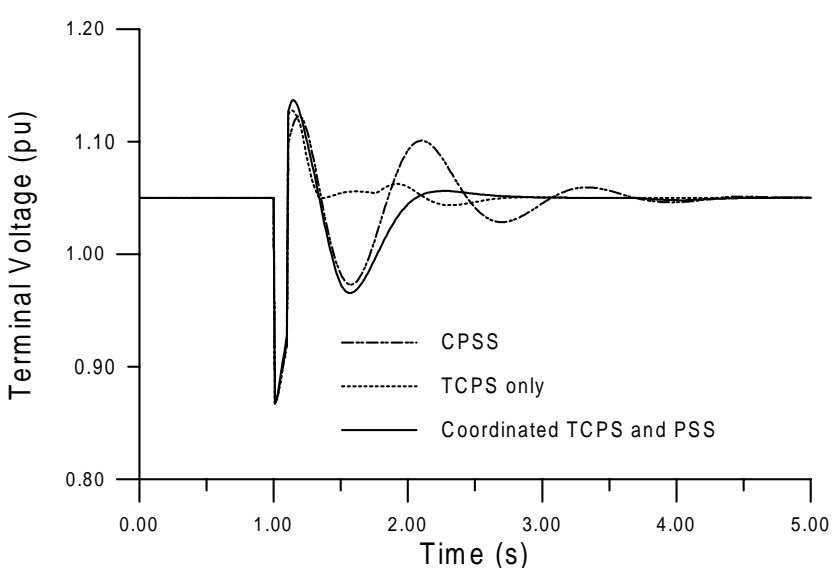

Fig. 9: Terminal voltage response with leading PF loading

\section{CONCLUSIONS}

In this study, the effects TCPS based stabilizer when applied independently and also through coordination with PSS have been investigated. The stabilizer design problem is formulated as an optimization problem where SA algorithm has been proposed to search for optimal settings of stabilizer parameters. The proposed control schemes have been tested on a weakly connected power system under different disturbances and loading conditions. The results show that

(a) the potential of SA algorithm to solve the problem of TCPS based stabilizer design;

(b) the TCPS based stabilizer provides good damping of low frequency oscillations and improves greatly the voltage profile;

(c) the effectiveness and robustness of the proposed control schemes over a wide range of loading conditions.

\section{ACKNOWLEDGEMENT}

The author would like to acknowledge the support of King Fahd University of Petroleum \& Minerals, Saudi Arabia. 


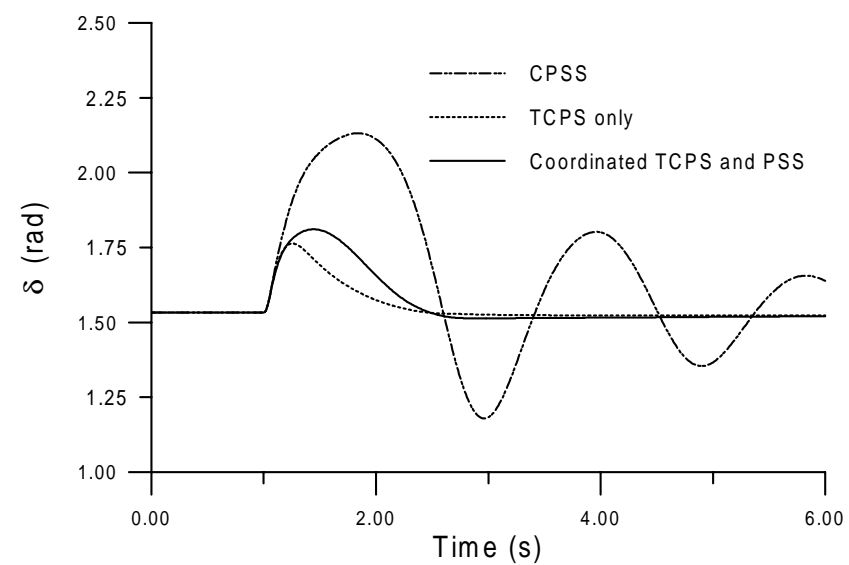

Fig. 10: $\delta$ response with heavy loading

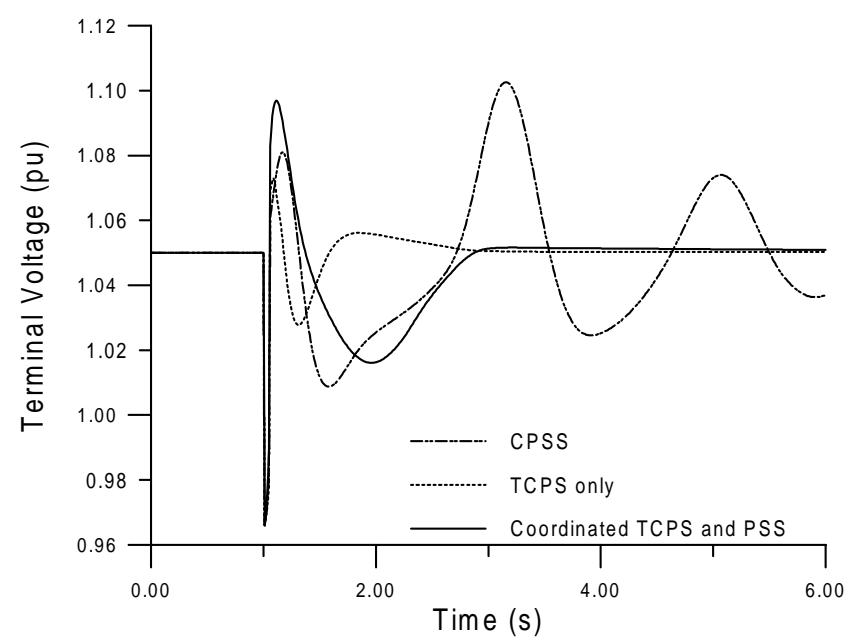

Fig. 11: Terminal voltage response with heavy loading

\section{REFERENCES}

[1] E. Larsen and D. Swann, "Applying Power System Stabilizers," IEEE Trans. PAS, Vol. 100, No. 6, 1981, pp. 3017-3046.

[2] G. T. Tse and S. K. Tso, "Refinement of Conventional PSS Design in Multimachine System by Modal Analysis," IEEE Trans. PWRS, Vol. 8, No. 2, 1993, pp. 598-605.

[3] P. Kundur, M. Klein, G. J. Rogers, and M. S. Zywno, "Application of Power System Stabilizers for Enhancement of Overall System Stability," IEEE Trans. PWRS, Vol. 4, No. 2, 1989, pp. 614-626.

[4] A. Rahim and S. Nassimi, "Synchronous Generator Damping Enhancement Through Coordinated control of Exciter and SVC," IEE Proc. Genet. Transm. Distrib., Vol. 143, No. 2, 1996, pp. 211-218.

[5] A. R. Mahran, B. W. Hogg, and M. L. Al-Sayed, "Coordinated Control of Synchronous Generator Excitation and Static VAR Compensator," IEEE Trans. Energy Conversion, Vol. 7, No. 4, 1992, pp. 615-622.

[6] H. F. Wang, F. J. Swift, and M. Li, "Analysis of Thyristorcontrolled Phase Shifter applied in Damping power system Oscillations," Int. J. Electrical Power \& Energy Systems, Vol. 19, No. 1, 1997, pp. 1-9.
[7] M. Iravani and D. Maratukulam, "Review of Semiconductor-Controlled (Static) Phase Shifters for Power System Applications," IEEE Trans. PWRS, Vol. 9, No. 4, 1994, pp. 1833-1839.

[8] A. Edris, "Enhancement of First-Swing Stability Using a High-Speed Phase Shifter," IEEE Trans. PWRS, Vol. 6, No. 3, 1991, pp. 1113-1118.

[9] F. Jiang, S. S. Choi, and G. Shrestha, "Power System Stability Enhancement Using Thyristor controlled phase shifter," IEEE Trans. PWRS, Vol. 12, No. 1, 1997, pp. 207214.

[10] Y. L. Tan and Y. Wang, "Nonlinear Excitation and Phase Shifter Controller for Transient Stability Enhancement of Power Systems Using Adaptive Control Law," Int. J. Electrical Power \& Energy Systems, Vol. 18, No. 6, 1996, pp. 397-403.

[11] E. Aarts and J. Korst, Simulated Annealing and Boltzmann Machines: A Stochastic Approach to Combinatorial Optimization and Neural Computing, John Wiley \& Sons, 1989.

[12] N. Metropolis, A. Rosenbluth, M. Rosenbluth, A. Teller, and E. Teller, "Equation of State Calculations by Fast Computing Machines," Journal of Chemical Physics, Vol. 21, 1953, pp. 1087-1092.

[13] Y. Y. Hsu and C. L. Chen, "Identification of Optimum Location for Stabilizer Applications Using Participation Factors," IEE Proc., Pt. C, Vol. 134, No. 3, May 1987, pp. 238-244.

[14] M. A. Abido, "A novel approach to conventional power system stabilizer design using tabu search," Accepted for Publication in Int. J. of Electrical Power \& Energy Systems, Oct. 1998.

[15] M. A. Abido, "A tabu search based approach to power system stability enhancement via excitation and static phase shifter control," Accepted for Publication in Electric Power Systems Research, Dec. 1998.

\section{BIOGRAPHY}

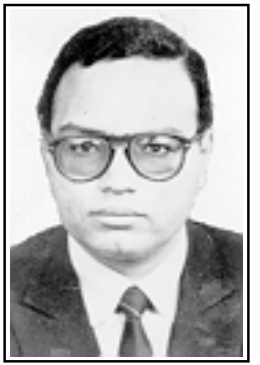

M. A. Abido received his B. Sc. (Honors with first class) and M. Sc. degrees in Electrical Engineering from Menoufia University, Egypt, in 1985 and 1989 respectively and $\mathrm{Ph}$. D degree from King Fahd University of Petroleum and Minerals, Saudi Arabia, in 1997.

He joined Electrical Engineering Dept., Menoufia University as a Graduate Assistant, as a Lecturer, and as an Assistant Professor during the periods of 1985-1989, 1989-1992, and 1998-present respectively. He joined KFUPM as a Lecturer from 1992-1997 where he is currently a Research Associate.

His research interests are power system planning, operation and stability, FACTS, system identification, optimization techniques applied to power systems, and intelligent control. 